\title{
Características de Vigas Laminadas Coladas Confeccionadas com Madeira de Teca (Tectona grandis)
}

\author{
Setsuo Iwakiri ${ }^{1}$, Jorge Luis Monteiro de Matos ${ }^{1}$, José Guilherme Prata ${ }^{1}$, \\ Rosilani Trianoski ${ }^{1}$, Carlos Frederico Parchen ${ }^{2}$, Vinicius Gomes Castro ${ }^{3}$, \\ Viviane Teixeira Iwakiri ${ }^{4}$
}

${ }^{1}$ Departamento de Engenharia e Tecnologia Florestal, Universidade Federal do Paraná - UFPR, Curitiba/PR, Brasil ${ }^{2}$ Departamento de Construção Civil, Universidade Federal do Paraná - UFPR, Curitiba/PR, Brasil ${ }^{3}$ Programa de Pós-Graduação em Engenharia Florestal, Universidade Federal do Paraná - UFPR, Curitiba/PR, Brasil ${ }^{4}$ Programa de Pós-Graduação em Arquitetura e Urbanismo, Universidade Federal de Santa Catarina - UFSC, Florianópolis/SC, Brasil

\begin{abstract}
RESUMO
O objetivo desta pesquisa foi avaliar a influência do adesivo, das gramaturas e dos tempos de prensagem nas propriedades mecânicas de vigas laminadas coladas, confeccionadas com madeira de teca (Tectona grandis). As vigas foram produzidas com cinco lamelas, sendo duas da classe de módulo de elasticidade dinâmico, classe 1 - MOEd C1 nas faces, e três da classe 2 - MOEd C2 no miolo. Para a colagem, utilizou-se o adesivo à base de resorcina-fenolformaldeído (RFF), com gramaturas de 350 e 450 g.m $\mathrm{m}^{-2}$, e tempos de prensagem de 5 e 8 horas. Para o adesivo à base de emulsão polimérica de isocianato (EPI), a colagem foi realizada com gramaturas de 180 e 220 g.m - $^{-2}$ e tempos de prensagem de 3 e 4 horas. Os resultados das propriedades mecânicas das vigas indicaram que não houve uma relação bem definida dos efeitos das variáveis de estudo nas propriedades de flexão estática. As vigas coladas com o adesivo RFF tiveram maior resistência da linha de cola em comparação às vigas coladas com o adesivo EPI.
\end{abstract}

Palavras-chave: resorcina-fenol-formaldeído, linha de cola, flexão estática.

\section{Characteristics of Glued Laminated Beams Made of Teak Wood (Tectona grandis)}

\begin{abstract}
This research aimed to evaluate the effects of different adhesives, amounts of glue, and pressing time on the mechanical properties of glued laminated beams (glulam) made of Tectona grandis wood. The beams were produced with 5 battens: two of dynamic modulus of elasticity, class 1 - MOEd C1, on the faces; and three of class 2 - MOEd C2, in the core. Gluing was performed with the use of resorcinol-phenol-formaldehyde (RPF) adhesive at the amounts of 350 and 450 g. $\mathrm{m}^{-2}$ and pressing time of 5 and 8 hours; and emulsion polymer isocyanate (EPI) adhesive at the amounts of 180 and $220 \mathrm{~g} . \mathrm{m}^{-2}$ and pressing time of 3 and 4 hours. The results of the mechanical properties of beams did not show a clear relationship of the effects of the studied variables on the evaluated properties of static bending. The beams glued with RFF adhesive presented higher resistance of the glue line compared with the beams glued with EPI adhesive.
\end{abstract}

Keywords: resorcinol-phenol-formaldehyde, glue line, static bending. 


\section{INTRODUÇÃO}

A teca (Tectona grandis) é uma espécie com ocorrência natural no subcontinente índico e no sudeste asiático. Os plantios florestais de teca no Brasil foram introduzidos na década de 1960, no Município de Cáceres, Estado de Mato Grosso, e hoje ocupam uma área de cerca de 50 mil hectares. As condições climáticas favoráveis, o solo de melhor fertilidade e os tratos silviculturais adequados contribuíram para reduzir o ciclo de produção de 80 anos, na região de origem, para apenas 25 anos na Região Centro-Oeste e Norte do país (Rech, 2009).

A madeira de teca é amplamente conhecida no mercado internacional por suas qualidades tecnológicas, sendo considerada moderadamente pesada, com densidade média de $0,65 \mathrm{~g} . \mathrm{cm}^{-3}$. As principais características da madeira de teca são a sua durabilidade, a estabilidade dimensional e a resistência natural ao ataque de fungos e insetos, além de desenho, cor e ausência de nós, o que faz desta espécie a madeira de folhosa mais valorizada do mundo (Rech, 2009).

Por causa das boas propriedades, a madeira de teca pode ser destinada às múltiplas aplicações nas áreas de construção civil e indústria moveleira. No mercado internacional, em consequência do seu preço elevado, o uso se restringe às aplicações mais nobres, como móveis finos, esquadrias de qualidade, pisos e decoração interior e exterior (lambris, lâminas faqueadas, construção naval no revestimento de convés de veleiros e iates). Já a madeira de plantios florestais, com pequeno diâmetro, proveniente de desbastes, é utilizada principalmente na forma de madeira serrada para produção de painel colado lateral (EGP), o qual é amplamente empregado na fabricação de móveis, portas e na decoração interna (Rech, 2009).

Ampliar o uso da madeira de teca, especialmente aquela obtida dos primeiros desbastes para fabricação de produtos de maior valor agregado (PMVA), é uma estratégia para valorização da madeira e para incentivar os plantios florestais desta espécie. A produção de EGP de teca vem se consolidando como matéria-prima na produção de móveis e decorações de interiores. Mais uma opção para uso da madeira de teca seria para produção de vigas laminadas coladas para uso estrutural. Estudos têm sido realizados sobre uso de madeiras de pinus e eucalipto para produção de vigas laminadas coladas; essas espécies, embora em pequena escala, já vêm sendo empregadas comercialmente.

$\mathrm{Na}$ montagem de vigas laminadas coladas, a classificação de lamelas para composição das faces externas melhora a sua resistência mecânica à flexão estática. Um dos métodos mais utilizados para esta finalidade é a aplicação de ondas de tensão para obtenção do módulo de elasticidade dinâmico (MOEd) e posterior definição das classes de resistência das lamelas.

Cunha (2007) utilizou método de classificação das lamelas por meio de aplicação de ondas acústicas e obteve melhores resultados de módulo de elasticidade (MOE) e módulo de ruptura (MOR) das vigas compostas com lamelas de maior MOEd nas faces e menor MOEd no miolo.

Em estudos com painéis de lâminas paralelas, também tem sido utilizado esse método para melhorar a sua resistência mecânica. Matos (1997) utilizou lâminas de Pinus taeda classificadas em classes de MOEd para produção de painéis de lâminas paralelas e obteve aumentos significativos nos resultados de MOE e de MOR, com a colagem de lâminas de classes superiores nas faces dos painéis. Pio (2002) utilizou lâminas de Eucalyputs grandis classificadas com a aplicação de ondas de tensão e obteve painéis de maior resistência à flexão estática com lâminas de classes superiores de MOEd nas faces dos painéis de lâminas paralelas.

A influência da gramatura e do tempo de prensagem na qualidade de colagem da madeira foi relatada por Marra (1992). Com relação ao tempo de prensagem, existe um tempo mínimo necessário para que ocorram as funções de mobilidade do adesivo na interface com a madeira e posterior secagem e cura do mesmo.

Com relação à gramatura, há uma dependência entre a porosidade da madeira e a viscosidade do adesivo, resultando em diferentes níveis de penetrabilidade deste nos poros da madeira; como consequência, pode-se gerar linha de cola faminta ou muito espessa. No primeiro caso, a resistência da colagem será comprometida e, no segundo caso, pode ocorrer derramemento do adesivo em excesso 
pelas bordas das juntas coladas, resultando em prejuízo quanto aos aspectos econômicos.

Quanto ao tipo de adesivo, a resorcina-fenolformaldeído (RFF) é a mais empregada para colagem de produtos de madeira que requerem alta resistência mecânica associada à sua durabilidade em ambientes externos. Em razão do seu alto custo, alguns tipos de adesivos alternativos formulados com base em poliuretano e isocianato têm sido testados na produção de painéis EGP e vigas laminadas coladas (Marra, 1992).

Perante o exposto, este trabalho teve como objetivo avaliar a massa específica aparente da madeira e a influência de dois diferentes tipos de adesivos, gramaturas e tempo de prensagem nas propriedades mecânicas de vigas de madeira laminada colada de teca (Tectona grandis).

\section{MATERIAL E MÉTODOS}

Foi utilizada, nesta pesquisa, madeira de teca (Tectona grandis L.f.) com 18 anos de idade, proveniente de plantios localizados no Município de Brasnorte, Estado do Mato Grosso. Foram coletadas aleatoriamente cinco árvores, a partir das quais foram seccionadas três toras com $3,0 \mathrm{~m}$ de comprimento, identificadas como base, meio e topo.
Os diâmetros médios das árvores (DAP) variaram de 26,7 a $33,1 \mathrm{~cm}$.

Da posição central de cada tora, foi retirado um pranchão com espessura de $75 \mathrm{~mm}$ para preparação das lamelas com espessura de $25 \mathrm{~mm}$, para manufatura de vigas laminadas coladas. Das extremidades das lamelas, foram retirados corpos de prova com as dimensões de $20 \times 30 \times 50 \mathrm{~mm}$ (largura $\times$ altura $\times$ comprimento), para determinação da massa específica aparente da madeira a $12 \%$ de teor de umidade. As etapas de preparação do material estão ilustradas na Figura 1.

As lamelas com dimensões nominais de $25 \times 75$ $\times 2.200 \mathrm{~mm}$ (espessura $\times$ largura $\times$ comprimento) foram secas ao ar livre e climatizadas até atingir o teor de umidade médio de $12 \%$. A seguir, foram aplainadas nas quatro faces para atingirem dimensões finais de $20 \times 60 \times 2200 \mathrm{~mm}$ (espessura $\times$ largura $\times$ comprimento).

As lamelas foram classificadas por meio da aplicação de ondas de tensões com uso do aparelho stress wave timer. Para a realização deste trabalho, foram utilizadas as lamelas classificadas como classe C1 com MOEd na faixa de 15.001 a 17.500 MPa nas faces, e classe C2 com MOEd na faixa de 12.5012 a 15.000 MPa no miolo das vigas. Esse procedimento foi adotado para padronizar e minimizar os efeitos
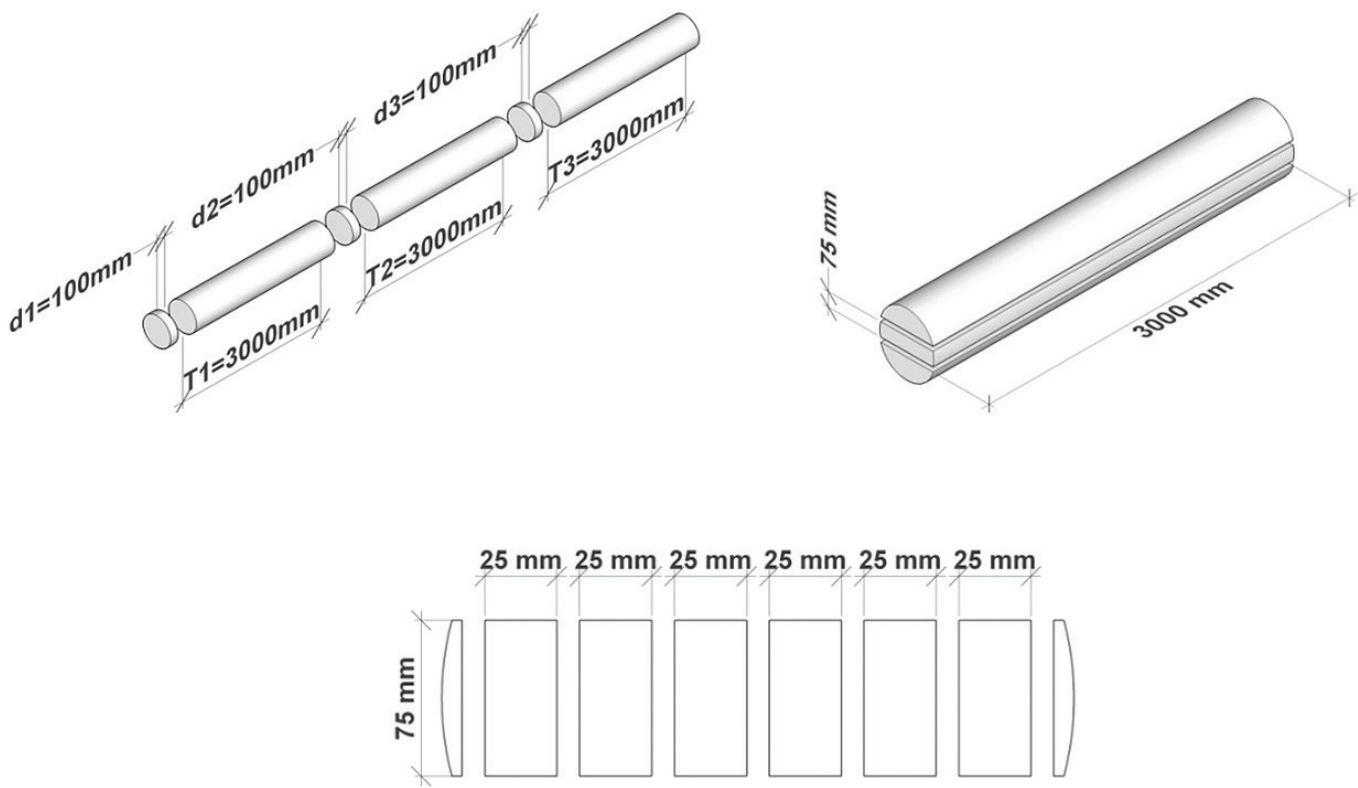

Figura 1. Esquema de obtenção das lamelas para manufatura das vigas. Figure 1. Schema to production of lamellas for glulam manufacture. 
da resistência das lamelas nas propriedades das vigas laminadas, e para possibilitar a avaliação das variáveis de estudos, tendo em vista que, devido às limitações de material, foi produzida apenas uma viga por tratamento.

As vigas foram produzidas com cinco camadas, com dimensões de $60 \times 100 \times 2.200 \mathrm{~mm}$ (largura $\times$ altura $\times$ comprimento), conforme o delineamento experimental apresentado na Tabela 1.

Para a colagem das vigas, foram utilizados os seguintes adesivos: 1) resorcina-fenol-formaldeído (RFF), com viscosidade Brookfield de $650 \mathrm{cP}$, teor de sólidos de 55\% e pH de 7,0;2) emulsão aquosa de poliuretano e isocianato (EPI) com viscosidade Brookfield de $5.500 \mathrm{cP}$, teor de sólidos de $44 \%$ e pH de 7,75 .

O RFF foi misturado com o endurecedor FM$316 \mathrm{M}$ nas proporções de 100 para 20 partes e o adesivo foi aplicado sobre uma das faces da lamela com gramatura de 350 e 450 g.m-2. Já para o EPI, o adesivo foi misturado com o endurecedor WA-742 nas proporções de 100 para 15 partes e o adesivo foi aplicado numa das faces da lamela com gramatura de 180 e 220 g.m - $^{-2}$

As vigas foram prensadas num aparato especial com grampos de aço com pressão específica de 1 MPa e tempo de prensagem de 5 e 8 horas para o RFF, e de 3 e 4 horas para o EPI, conforme descrito no delineamento experimental. A variação da temperatura ambiente no período de prensagem das vigas foi de 20,5 a $27,8^{\circ} \mathrm{C}$.

Após a prensagem, as vigas foram acondicionadas em câmara climática com temperatura de $20 \pm 2{ }^{\circ} \mathrm{C}$ e umidade relativa de $65 \pm 5 \%$, até atingir a umidade de equilíbrio em torno de $12 \%$. Os ensaios de flexão estática foram realizados em máquina universal de ensaios de acordo com a American Socitey for Testing and Materials - ASTM D-198 (ASTM, 1996), para determinação de módulo de elasticidade (MOE) e módulo de ruptura (MOR), sendo utilizada uma viga por tratamento. A avaliação da resistência da linha de colagem foi realizada por meio dos ensaios de cisalhamento com base na ASTM D-3110 (ASTM, 1990), em oito corpos de prova retirados das extremidades de cada viga.

Os resultados de flexão estática das vigas foram analisados pela comparação dos valores individuais de MOE e MOR, obtidos para cada viga, tendo em vista que a quantidade de lamelas foi insuficiente para produzir vigas com repetições para cada tratamento. Para os ensaios de cisalhamento da linha de cola, os resultados foram analisados por meio de análise de variância, ao empregar um delineamento inteiramente casualizado; note-se que, quando o efeito do tratamento foi significativo, as médias foram comparadas pelo teste de Tukey, em nível de probabilidade de $95 \%$.

\section{RESULTADOS E DISCUSSÕES}

\subsection{Massa específica aparente}

O valor médio da massa específica aparente da madeira de teca foi de $0,519 \mathrm{~g} . \mathrm{cm}^{-3}$. Verificou-se uma redução nos valores de massa específica no sentido base-topo da árvore de 0,550 para 0,522 g.cm $\mathrm{gm}^{-3}$, e aumento no sentido medula-casca de 0,456 para $0,558 \mathrm{~g} \cdot \mathrm{cm}^{-3}$.

Tabela 1. Delineamento experimental para manufatura das vigas com diferentes adesivos, gramaturas e tempos de prensagem.

Table 1. Experimental design to manufacture of beams with different kinds and amount of adhesives and press time.

\begin{tabular}{|cccc|}
\hline Tratamento & Adesivo & Gramatura (g.m $\mathbf{~}^{-2}$ & Tempo de prensagem (hora) \\
\hline T1-RFF/350/5 & & 350 & 5 \\
\hline T2-RFF/350/8 & RFF & & 8 \\
\hline T3-RFF/450/5 & & 450 & 5 \\
\hline T4-RFF/450/8 & & 180 & 3 \\
\hline T5-EPI/180/3 & EPI & & 4 \\
\hline T6-EPI/180/4 & & 220 & 3 \\
\hline T7-EPI/220/3 & & & 4 \\
\hline T8-EPI/220/4 & & & 3 \\
\hline
\end{tabular}

RFF: resorcina-fenol-formaldeído; EPI: emulsão aquosa de poliuretano e isocianato. 
Os valores obtidos foram inferiores em relação à massa específica básica de teca citados por Melo et al. (2006), na faixa de $0,590 \mathrm{~g} / \mathrm{cm}^{3}$ a $0,630 \mathrm{~g} \cdot \mathrm{cm}^{-3}$. Santos (2010) obteve, para madeira juvenil de teca com 7 anos de idade, densidade básica média de 0,450 g. $\mathrm{cm}^{-3}$. Lima et al. (2011) avaliaram a densidade aparente da madeira de teca com 32 anos de idade, coletada no Município de Pederneiras, Estado de São Paulo, e obtiveram valor médio de 0,640 g. $\mathrm{cm}^{-3}$ e aumento na direção medula-casca da árvore.

Com relação às outras espécies provenientes de plantios florestais de interesse comercial, Trianoski (2012) obteve, para sete espécies de pinus tropicais da região de Ventania - PR, com 18 anos de idade, massa específica básica de 0,431 g.cm ${ }^{-3}$ a 0,560 g.cm${ }^{3}$. Silva (2004) obteve, para madeiras de Eucalyptus grandis provenientes de plantios com 10, 14, 20 e 25 anos, massa específica de 0,340,0,410,0,470 e 0,420 g. $\mathrm{cm}^{-3}$, respectivamente. Embora numericamente a massa específica básica seja um pouco inferior em relação à massa específica aparente a $12 \%$ de umidade, os valores comparativos demonstram que a madeira de teca possui massa específica na mesma faixa de pinus tropicais e um pouco superior em relação à madeira de Eucalyptus grandis.

\subsection{Ensaio de flexão estática}

Na Tabela 2, estão apresentados os resultados de módulo de elasticidade e módulo de ruptura em flexão estática das vigas laminadas coladas, produzidas com adesivos RFF e EPI, com duas diferentes gramaturas e tempos de prensagem.

O menor valor de MOE foi obtido para viga produzida com adesivo RFF, com gramatura de 450 g. $\mathrm{m}^{-2}$ e tempo de prensagem de 8 horas, e o maior, para viga produzida com adesivo EPI, com gramatura de 220 g.m $\mathrm{m}^{-2}$ e tempo de prensagem de 3 horas. Já para o MOR, o menor valor foi obtido para viga produzida com adesivo RFF, com gramatura de 450 g.m $\mathrm{m}^{-2}$ e tempo de prensagem de 5 horas, e o maior valor foi obtido para viga produzida com adesivo EPI, com gramatura de 180 g.m $\mathrm{m}^{-2}$ e tempo de prensagem de 3 horas. Portanto, os maiores valores de MOE e MOR foram obtidos para as vigas produzidas com adesivo EPI.

Os resultados obtidos nesta pesquisa estão compatíveis com alguns valores encontrados na literatura. Cunha (2007) obteve, para vigas de Pinus taeda coladas com resina resorcina-fenolformaldeído, MOE de 15.589 MPa e MOR de 26 MPa. Nielsen (1998) obteve, para vigas produzidas com madeira de Eucalyptus grandis com 9 anos de idade, MOE de $10.885 \mathrm{MPa}$ e MOR de $41 \mathrm{MPa}$. Os resultados de MOR obtidos para as vigas de teca foram altamente satisfatórios em comparação aos valores referenciados para vigas de Pinus taeda e de Eucalyptus grandis.

Para as vigas produzidas com o adesivo RFF, o aumento na gramatura de 350 para 450 g.m ${ }^{-2}$ influenciou de forma positiva apenas no MOE da viga prensada com tempo de 5 horas e no MOR da viga prensada com tempo de 8 horas. Para as vigas produzidas com o adesivo EPI, o aumento na gramatura de 180 para 220 g.m ${ }^{-2}$ resultou em maior $\mathrm{MOE}$ das vigas prensadas com 3 horas. $\mathrm{O}$ aumento na gramatura reduziu os valores de MOR para os dois tempos de prensagem, o que indica a possibilidade de redução na quantidade de adesivo e, consequentemente, no custo final da produção de vigas.

Para as vigas produzidas com o adesivo RFF, o aumento no tempo de prensagem resultou em

Tabela 2. Efeitos dos adesivos, gramaturas e tempos de prensagem nos módulos de elasticidade e de ruptura.

Table 2. Effects of adhesives, amounts and press time in the modulus of elasticity and rupture.

\begin{tabular}{|c|c|c|c|c|c|}
\hline Tratamento & Adesivo & Gramatura (g.m $\left.\mathrm{m}^{-2}\right)$ & Tempo prensagem (h) & MOE (MPa) & MOR (MPa) \\
\hline $\mathrm{T} 1$ & \multirow{4}{*}{ RFF } & \multirow{2}{*}{350} & 5 & 12.570 & 66 \\
\hline $\mathrm{T} 2$ & & & 8 & 12.893 & 45 \\
\hline T3 & & \multirow{2}{*}{450} & 5 & 13.675 & 38 \\
\hline $\mathrm{T} 4$ & & & 8 & 12.405 & 83 \\
\hline T5 & \multirow{4}{*}{ EPI } & \multirow{2}{*}{180} & 3 & 12.687 & 85 \\
\hline T6 & & & 4 & 13.506 & 56 \\
\hline T7 & & \multirow{2}{*}{220} & 3 & 14.274 & 43 \\
\hline T8 & & & 4 & 12.817 & 50 \\
\hline
\end{tabular}


Tabela 3. Efeitos dos adesivos, gramaturas e tempos de prensagem na resistência da linha de cola.

Table 3. Effects of adhesives, amounts and press time in the glue line shear strength.

\begin{tabular}{ccccc} 
Adesivo & Gramatura $\left(\mathbf{g} \cdot \mathbf{m}^{-2}\right)$ & Tempo $(\mathbf{h})$ & Média (MPa) & Falhas (\%) \\
\multirow{2}{*}{ RFF } & \multirow{2}{*}{350} & 5 & $13,27 \mathrm{e}$ & 56,25 \\
& \multirow{2}{*}{450} & 8 & $12,44 \mathrm{de}$ & 42,50 \\
& \multirow{2}{*}{180} & 5 & $14,17 \mathrm{e}$ & 51,25 \\
& \multirow{2}{*}{ EPI } & 8 & $12,02 \mathrm{cde}$ & 38,75 \\
\hline & \multirow{2}{*}{220} & 4 & $6,73 \mathrm{a}$ & 31,43 \\
& 3 & $9,38 \mathrm{~b}$ & 28,60 \\
\hline
\end{tabular}

pequeno incremento do $\mathrm{MOE}$ das vigas coladas com gramatura de 350 g.m $\mathrm{m}^{-2}$. O aumento no tempo de prensagem resultou em maior valor de MOR da viga produzida com gramatura de 450 g. $\mathrm{m}^{-2}$. Para as vigas produzidas com o adesivo EPI, o aumento no tempo de prensagem contribuiu para o incremento no valor de $\mathrm{MOE}$ da viga colada com gramatura de 180 g. $\mathrm{m}^{-2}$, e do MOR da viga colada com gramatura de 220 g. $\mathrm{m}^{-2}$.

As avaliações gerais dos resultados de MOE e MOR demonstram que não há uma relação bem definida quanto aos efeitos de gramatura e tempo de prensagem sobre estas propriedades. Considerandose a importância da resistência das vigas para uso estrutural, o melhor resultado obtido entre as vigas produzidas com adesivo RFF foi para a colagem realizada com a gramatura de 450 g.m ${ }^{-2}$ e o tempo de prensagem de 8 horas. Entre as vigas produzidas com adesivo EPI, o melhor resultado foi obtido para a colagem realizada com gramatura de 180 g.m $\mathrm{m}^{-2}$ e tempo de prensagem de 3 horas.

\subsection{Cisalhamento da linha de cola}

$\mathrm{Na}$ Tabela 3, estão apresentados os resultados da resistência ao cisalhamento da linha de cola das vigas laminadas coladas, produzidas com adesivos RFF e EPI, com duas diferentes gramaturas e tempos de prensagem.

Os valores médios de resistência da linha de cola ao cisalhamento variaram de 12,02 a 14,17 MPa para as vigas produzidas com adesivo RFF, e de 6,73 a 10,39 MPa para aquelas confeccionadas com adesivo EPI. Não foram constatadas diferenças estatisticamente significativas entre as médias obtidas para as vigas coladas com RFF, indicando que não houve influência da gramatura e do tempo de prensagem na resistência da linha de cola.

Já para as vigas coladas com EPI, o tratamento com menor gramatura (180 g.m $\left.{ }^{-2}\right)$ e tempo de prensagem $(3 \mathrm{~h})$ exibiu média estatisticamente inferior em comparação aos demais tratamentos. $\mathrm{O}$ aumento no tempo de prensagem não influenciou significativamente na resistência da linha de cola das vigas coladas com gramatura de 220 g.m ${ }^{-2}$. Entretanto, para aquelas prensadas com tempo de 3 horas, o aumento na gramatura de 180 para 220 g.m${ }^{2}$ resultou em aumento significativo da resistência da linha de cola. De forma geral, as vigas coladas com adesivo RFF tiveram maiores valores de resistência da linha de cola.Os valores de percentagens de falhas na madeira variaram na faixa de $26,25 \%$ a $56,25 \%$.

Vital et al. (2006) encontraram, para juntas coladas de madeira de Eucalyptus saligna, Eucalyptus grandis e Pinus elliottii com adesivo resorcina-fenolformaldeído, valores de tensões de cisalhamento e percentagens de falhas na madeira de 9,62 $\mathrm{MPa}$ e 29,73\%; 8,05 MPa e 41,42\%, e 5,63 MPa e 53,25\%, respectivamente. Lima et al. (2008) obtiveram, para ensaios de cisalhamento da linha de cola para madeiras de quatro clones de Eucalyptus, resultados de tensões de cisalhamento e percentagens de falhas na madeira na faixa de 7,55 a 9,07 $\mathrm{MPa}$ e $29 \%$ a $64 \%$, respectivamente. Portanto, os resultados obtidos nesta pesquisa são satisfatórios em comparação aos valores obtidos pelos referidos autores.

\section{CONCLUSÕES}

Os resultados de propriedades mecânicas obtidas para as vigas laminadas coladas confeccionadas com a madeira de teca foram satisfatórios em comparação 
aos valores contidos na literatura para madeiras de pinus e eucalipto, utilizadas como referência.

Não foram constatadas relações bem definidas dos efeitos do tipo de adesivo (RFF, EPI), das diferentes gramaturas e dos tempos de prensagem sobre o MOE e o MOR das vigas.

A gramatura e o tempo de prensagem não influenciaram nos resultados de resistência da linha de cola das vigas produzidas com adesivo RFF. Para as vigas coladas com adesivo EPI, com gramatura de 180 g. $\mathrm{m}^{-2}$, o aumento no tempo de prensagem resultou em maior resistência da linha de cola.

As vigas coladas com o adesivo RFF apresentaram maior resistência da linha de cola em comparação às vigas coladas com o adesivo EPI; entretanto, estes resultados não influenciaram na resistência estrutural das vigas laminadas coladas.

\section{AGRADECIMENTOS}

Agradecimentos às Empresas Berneck Painéis e Serrados S.A., e Momentive Ind. Química S.A., pelas doações de madeira e adesivos, respectivamente. Ao $\mathrm{CNPq}$, pelo apoio financeiro para o desenvolvimento desta pesquisa.

\section{STATUS DA SUBMISSÃO}

Recebido: 31 out., 2012

Aceito: 17 ago., 2013

Publicado: 30 jun., 2014

\section{AUTOR(ES) PARA CORRESPONDÊNCIA}

\section{Setsuo Iwakiri}

Departamento de Engenharia e Tecnologia

Florestal, Universidade Federal do

Paraná - UFPR, CEP 80210-170, Curitiba, PR,

Brasil

e-mail: setsuo@ufpr.br

\section{REFERÊNCIAS}

American Society for Testing and Materials - ASTM. ASTM D198: Static tests of timbers in structural sizes. Philadelphia; 1996.

American Society for Testing and Materials - ASTM. ASTM D3110: Standard specification for adhesives used in nonstructural glued lumber products. Philadelphia; 1990.

Cunha AB. Produção de vigas estruturais de madeira avaliadas por meio de ensaios estáticos e dinâmicos [tese]. Curitiba: Universidade Federal do Paraná; 2007.

Lima CKP, Mori FA, Mendes LM, Trugilho PF, Mori CLSO. Colagem da madeira de clones de Eucalyptus com três adesivos comerciais. Scientia Forestalis 2008; 36(77): 73-77.

Lima IL, Pimentel MM, Garcia JN. Propriedades mecânicas e densidade aparente da madeira de Tectona grandis L.f. (Teca) em função do espaçamento e da posição radial na tora. Silva Lusitana 2011; 19(2): 221 232.

Marra AA. Technology of wood bonding: principles and practice. New York: Van Nostrand Reinhold; 1992.

Matos JLM. Estudos sobre a produção de painéis estruturais de lâminas paralelas de Pinus taeda L. [tese]. Curitiba: Universidade Federal do Paraná; 1997.

Melo RR, Paes JB, Lima CR, Ferreira, AG. Estudo da variação radial da densidade básica de sete madeiras do semi-árido. Revista Científica Eletrônica de Engenharia Florestal 2006; 4(7): 1-8.

Nielsen IR. Utilização da madeira comercial do híbrido de Eucalyptus urophylla S.T. Blake X Eucalyptus grandis Hill ex Maiden na confecção de vigas laminadas coladas [dissertação]. Curitiba: Universidade Federal do Paraná; 1998.

Pio NS. Produção de painéis estruturais de laminas paralelas (PLP) de Eucalyptus grandis Hill ex Maiden. Curitiba: Universidade Federal do Paraná; 2002. PMCid:PMC2567543

Rech C. Características da teca. Revista da Madeira 2009; (118): 55-57.

Santos JL. Caracterização tecnológica da madeira juvenil de Tectona grandis L.f. visando a produção de móveis [monografia]. Jerônimo Monteiro: Universidade Federal do Espírito Santo; 2010.

Silva JC, Oliveira JTS, Matos JLM, Tomazello M Fo, Keinert S Jr. Influência da idade e da posição radial na massa específica da madeira de Eucalyptus grandis Hill ex. Maiden. Floresta 2004; 34(1): 13-22.

Trianoski R. Avaliação da qualidade da madeira de espécies de pinus tropicais por meio de métodos convencionais e não destrutivos [tese]. Curitiba: Universidade Federal do Paraná; 2012.

Vital BR, Maciel AS, Lucia RMD. Qualidade de juntas coladas com lâminas de madeira oriundas de três regiões do tronco de Eucalyptus grandis, Eucalyptus saligna e Pinus elliottii. Revista Árvore 2006; 30(4): 637-644. http://dx.doi.org/10.1590/S0100-67622006000400017 